\section{Self-assembling organic nanotubes based on a cyclic peptide architecture}

\author{
M. Reza Ghadiri, Juan R. Granja, \\ Ronald A. Milligan, Duncan E. McRee \\ \& Nina Khazanovich
}

Nature 366, 324-327 (1993)

IN this Letter important references to earlier works were inadvertently left out. De Santis et al., in the context of a theoretical conformational analysis of linear regular L,D polypeptides, suggested formation of parallel and antiparallel cylindrical structures through interannular association of $\beta$-type rings ${ }^{1}$. Synthesis of some cyclic oligopeptides with $S_{2 n}$ symmetry, with the construction of tubular structures as the stated goal, was first reported by Tomasic and Lorenzi ${ }^{2}$, when the possibility oftubular aggregate formation was tentatively assigned based on the solubility profiles and infrared analysis ${ }^{2}$. We thank G. P. Lorenzi for kindly bringing these references to our attention.

1. De Santis, P., Morosetti, S. \& Rizzo, R. Macromolecules 7, 52-58 (1974).

2. Tomasic, L. \& Lorenzi, G. P. Helv. chim. Acta 70, 1012-1016 (1987).

\section{A deep earthquake aftershock sequence and implications for the rupture mechanism of deep earthquakes}

\section{Douglas A. Wiens, Jeffrey J. McGuire, Patrick J. Shore, Michael G. Bevis, Kitione Draunidalo, Gajendra Prasad \& Saimone P. Helu}

Nature 372, 540-543 (1994).

THE numbers on the depth scale in Fig. $3 a$ and $b$ in this Letter were incorrect, making the total width of the aftershock zone look as though it was $25 \mathrm{~km}$, rather than $50 \mathrm{~km}$ as correctly reported in the text. The numbers on the $y$-axis of Fig. $3 a$ should read 555, 575 and $595 \mathrm{~km}$ (not 565,575 and $585 \mathrm{~km}$ as published); on the $y$-axis of Fig. $3 b$ they should be $545,565,585$ and $605 \mathrm{~km}$ (instead of 560, 570, 580 and $590 \mathrm{~km}$ ).

\section{A TCP1-related molecular chaperone from plants refolds phytochrome to its photoreversible form}

\section{Eckart Mummert, Rudolf Grimm, Volker Speth, Christoph Eckerskorn, Emile Schiltz, Anthony A. Gatenby \& Eberhard Schäfer}

Nature 363, 644-648 (1993)

THE following correction concerns only the contribution to this paper of E.M., R.G. and E. Schäfer. We purified a protein of $M_{\mathrm{r}} 60,000(60 \mathrm{~K})$ from oat seedlings that possesses an oligomeric structure of $600 \mathrm{~K}$ and is partially similar to the cytosolic TCP1 proteins from certain eukaryotes. Specific oligonucleotides were generated following internal peptide sequencing and were used to screen a cDNA library from oats. Ten clones were characterized by sequencing, all of them containing the TCP1-related internal sequenced published before our paper. The clones belong to two subclasses which mainly differ in the deduced amino-terminal sequence of the mature proteins: ALESA for one and ELESG for the other class.

All clones are very similar to $\beta$-glucosidase from Zea mays and glucosidases and galactosidases from other organisms ${ }^{1}$, but show no overall homology to TCP1 genes. The purified $60 \mathrm{~K}$ protein has $\beta$-glucosidase and $\beta$-galactosidase activity, which was recently confirmed by Rüdiger and co-workers ${ }^{2}$.

To test the putative chaperone activity of the $60 \mathrm{~K}$ protein, we purified it in high amounts using a purification procedure slightly different from that published in our paper. In contrast to the data published in our paper, this purified fraction is not able to refold phytochrome to its photoreversible form. On the other hand, it can prevent the aggregation of denatured citrate synthase in a light-scattering assay. Because the purified fraction contains traces of an $800 \mathrm{~K}$ protein strongly crossreacting with antibodies raised against chloroplast cpn60 or against $E$. coli GroEL, we cannot definitely exclude that the apparent chaperone activity is caused by the contaminating proteins and not by the $60 \mathrm{~K} \beta$-glucosidase.

1. Brzobohaty, B. et al. Science 262, 1051-1054 (1993).

2. Gus-Mayer, S. et al. FEBS Lett. 347, 51-54 (1994). 